\title{
FEATURED CASE REPORT
}

\section{Acute type A aortic dissection in a cardiac allograft recipient: case report and review of the literature}

\author{
C Schellemans, W Tack, M Vanderheyden
}

Heart 2004;90:1256-1258. doi: 10.1136/hrt.2003.024307

Dissection of the ascending aorta is uncommon in cardiac allograft recipients and usually affects the native aorta. The diagnosis is often made at necropsy. The first case is reported of a Bentall procedure performed in a heart transplant recipient who presented with ascending aortic dissection of the donor aorta and concomitant aortic regurgitation seven years after orthotopic transplantation.

$\mathrm{D}$ issection of the ascending aorta is uncommon in cardiac allograft recipients, usually affects the native aorta, and is often diagnosed at necropsy. One case report describes an aortic dissection confined to the donor aorta, which was successfully treated with a Hemashield graft. We report the first Bentall procedure performed in an orthotopic heart transplant recipient who presented with ascending aortic dissection of the donor aorta and concomitant aortic regurgitation seven years after orthotopic transplantation.

\section{CASE REPORT}

A 68 year old white man was admitted with progressive shortness of breath and thoracic pain. He had been well until two days before when he experienced thoracic pain, irradiating to the back. The pain was accompanied by exertional dyspnoea as well as by a non-productive cough. He underwent an orthotopic heart transplantation seven years before for end stage heart failure caused by ischaemic heart disease. At that time he received a heart from a 40 year old man with hypertension who had died of intracerebral bleeding. A habitus of Marfan of the donor was not reported. The length of the donor aorta extended $3 \mathrm{~cm}$ above the commissural edge and the recipient aorta was anastomosed by a Blalock 4-0 Prolene suture.

After this procedure his clinical condition normalised and no life threatening episodes of rejection occurred under immunosuppression with cyclosporine and prednisolone. Because of persistent arterial hypertension ranging from $145 / 90$ to $175 / 95 \mathrm{~mm} \mathrm{Hg}$, antihypertensive treatment with angiotensin converting enzyme inhibitors was started.

On admission, clinical examination showed a man in respiratory distress with increased jugular venous pulse and rales over both lung fields. Blood pressure was 140/85 mm Hg. At the base of the heart a new diastolic grade 4/6 murmur of aortic regurgitation was heard. The ECG was unchanged from previous tracings and showed a normal sinus rhythm at a rate of 100 beats/min together with an incomplete right bundle branch block.

On chest radiography the cardiothoracic index was augmented and pulmonary oedema was present. The mediastinum was widened.

Transthoracic echocardiography showed a non-dilated well contracting left ventricle. There was aortic regurgitation of grade $3 / 4$ with a pressure half time of $215 \mathrm{~ms}$ on pulsed wave Doppler because of prolapse of the non-coronary cusp.

The aortic root was dilated from $2.7 \mathrm{~cm}$ at time of implant to $5.1 \mathrm{~cm}$ and an intimal tear of the ascending aorta was noted. Aortic dissection type A with secondary aortic regurgitation was tentatively diagnosed and subsequently confirmed by transoesophageal echocardiography and magnetic resonance imaging (figs 1 and 2).

Surgical inspection showed aneurysmal dilatation of the ascending donor aorta without rupture. Both coronary arteries were dissected, as well as the non-coronary cusp of the aortic valve. Distally the dissection extended to the suture line with the recipient aorta. On the basis of these findings, a
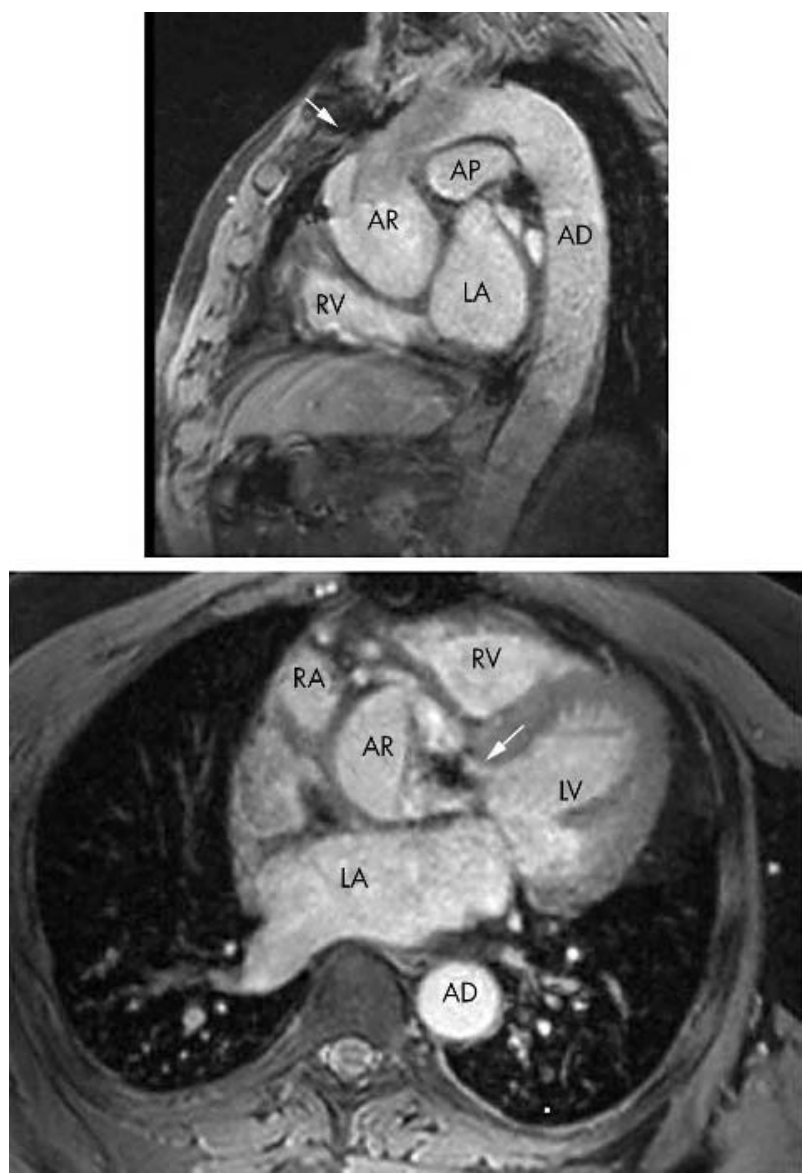

Figure 1 (Top) Magnetic resonance image in the sagittal axis of the heart. $A D$, descending aorta; $A P$, pulmonary artery; $A R$, aortic root; $L A$, left atrium; RV, right ventricle. The arrow indicates aneurysmal dilatation of the ascending aorta. (Bottom) Magnetic resonance image in the transverse axis of the heart. RA, right atrium. The arrow indicates an aortic valve regurgitation jet. 


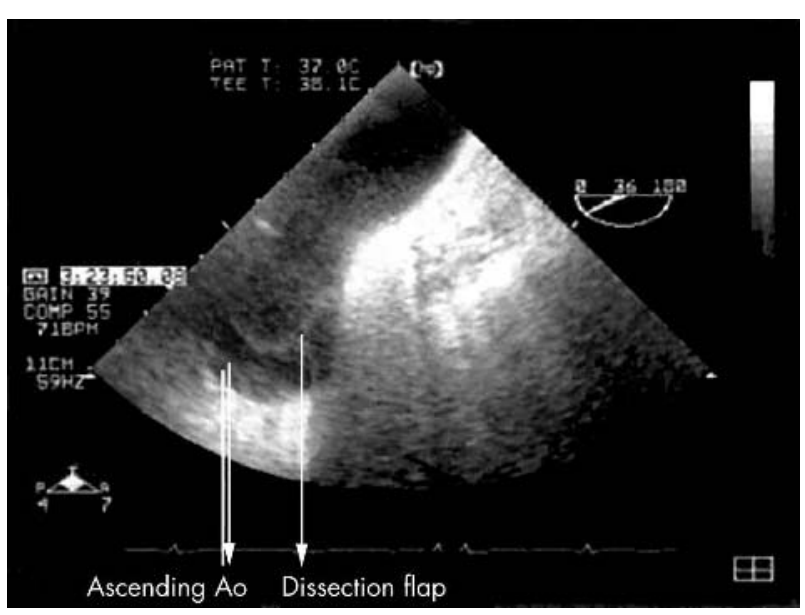

Figure 2 Transoesophageal echocardiograph in the long axis showing the dissection flap of the recipient ascending aorta (Ao).

Bentall procedure was performed. The aorta was reconstructed with a Hemashield graft that was sutured to the recipient aorta at the previous suture location between the recipient and donor aorta.

An aortic valve bioprosthesis was inserted (Shellhigh 25) and the coronary ostia were reimplanted at the conduit. The patient was weaned from cardiopulmonary bypass with minimal inotropic support. He recovered gradually and was discharged from the hospital in good condition on day 28.
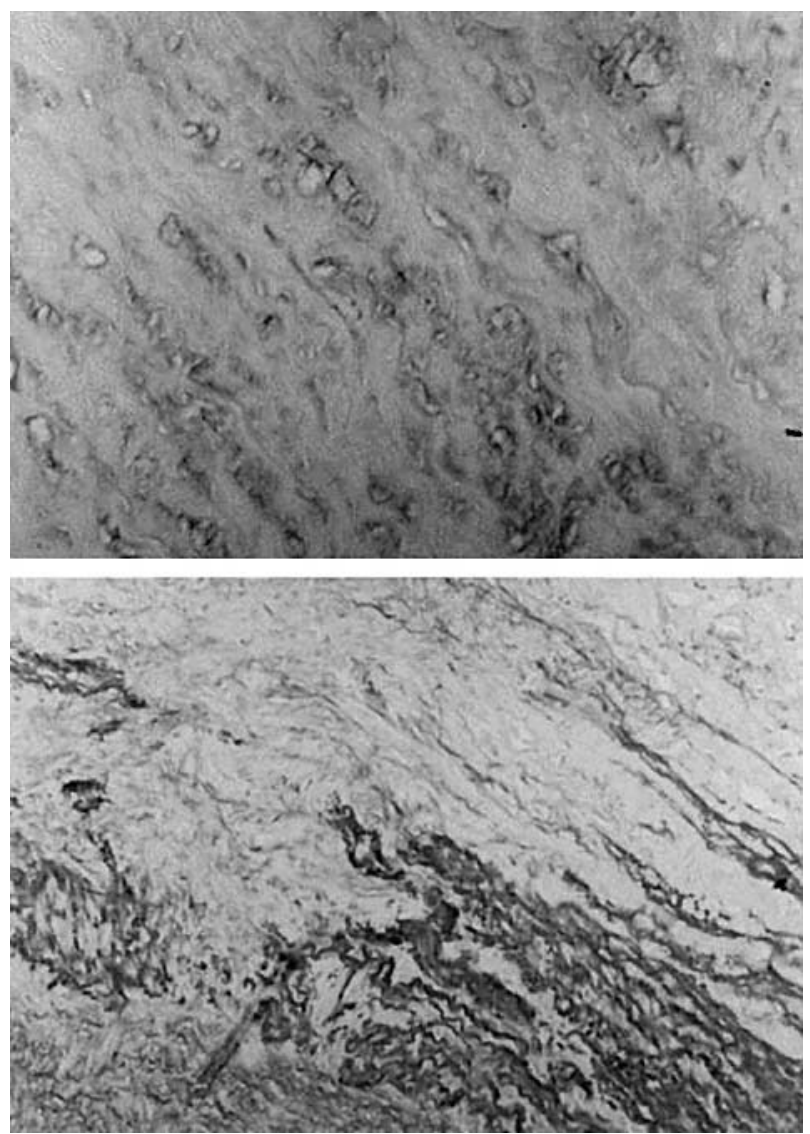

Figure 3 Microscopical examination. (Top) Cystic medial necrosis together with deposits of alcian blue positive mucopolysaccharides (alcian blue, magnification $\times 200$ ). (Bottom) Elastic fragmentation in the separated layers of the dissection (elastica, magnification $\times 200$ ).
Cyclosporine $(2 \times 100 \mathrm{mg} /$ day $)$ and prednisolone $(5 \mathrm{mg} /$ day $)$ were continued as immunosuppression. Heart catheterisation performed six months after the event at the annual examination found normal left ventricular function with no evidence for graft vasculopathy or residual aortic regurgitation.

Histological examinations showed typical alterations of cystic media necrosis, such as focal loss of muscle cells together with deposits of alcian blue positive mucopolysaccharides (fig 3), elastic fragmentation (fig 3), fibrosis, and cystic medial necrosis, and no inflammation. The aortic leaflets had mucoid degeneration and were not calcified.

\section{DISCUSSION}

Heart transplantation has become a highly successful treatment option for patients with end stage heart failure that is unresponsive to standard medical and surgical treatment. Satisfactory long term survival is achieved with overall seven year survival rates exceeding $74 \% .{ }^{1}$ Because of the simplicity of the procedure, surgical complications related to orthotopic heart transplantation are relatively rare: atrial thrombosis at the suture line, ${ }^{2}$ atrial distortion with consequent valve incompetence, ${ }^{34}$ pressure gradient in a distorted pulmonary anastomosis, ${ }^{5}$ creation of a pseudo-cor triatum, ${ }^{67}$ and prolonged sinoatrial block ${ }^{8}$ have all been described.

Thanks to new techniques the incidence of these complications is decreasing even further. Aortic dissection has been reported after renal and heart transplantation. Its frequency after orthotopic heart transplantation is extremely rare, varying between $1-2 \%$. Usually the native aorta is affected. Involvement of the donor aorta has been reported in one case. $^{9}$

According to the underlying pathophysiology and the time of onset, patients can be categorised into four groups. The first group of patients presents with acute aortic rupture occurring in the early postoperative phase. This dramatic event results from a mismatch between the donor and recipient aorta. As a consequence the donor aorta is stretched, extensive tension is generated at the suture line, and the aorta will finally rupture. Careful alignment and trimming of donor and recipient aorta can prevent this complication.

The second group comprises patients with postoperative infections resulting in mediastinitis and mycotic or bacterial pseudoaneurysms of the ascending aorta.

The third group consists of patients with true aneurysms and dissection of the native aorta. The high incidence of arterial hypertension, diabetes mellitus, and accelerated arteriosclerosis caused by immunosuppression predisposes heart transplantation patients to this complication. Of note, accelerated growth of true atherosclerotic aneurysms of the ascending aorta, ${ }^{10}$ increased growth of abdominal aortic aneurysms, ${ }^{11}$ and increased incidence of perivascular disease ${ }^{12}$ have all been reported in heart transplant recipients.

The last group comprises aortic dissection in heterotopic heart transplant recipients. In these patients the aortic tear originates at the anastomosis where a limited portion of the native aorta, adjacent to the anastomosis, is subjected to high shear stresses.

The exact mechanism responsible for the dissection in our patient is not clear. Our patient did not have a history of infection, nor was there any evidence of connective tissue disease. Similar to Coppola and colleagues, ${ }^{13}$ we found evidence of chronic degeneration of the aortic wall with notable thinning and fibrosis of the intima. Bull and colleagues $^{12}$ observed a higher incidence of abdominal aneurysms after heart transplantation in patients older than 55 years with ischaemic cardiomyopathy and a preoperative ejection fraction below $20 \%$. They speculated that the better 
haemodynamic function may contribute to the development of these aneurysms. On the basis of these observations we assume that arteriosclerosis is the most plausible cause of aortic dissection in our patient.

Interestingly the dissection was limited to the donor aorta and did not extend in the native aorta. Similar observations have been reported and suggest that the aortic suture line can prevent progression of the dissection. ${ }^{9}$

Since infection, rejection, and transplant vasculopathy constitute the bulk of fatal illness among cardiac allograft recipients, aortic dissection is often underdiagnosed. Dissection may mimic various pathological states including myocardial infarction, heart failure, cardiogenic shock, and tamponade. As the heart is denervated, myocardial or aortic injury can also occur without pain and mediastinal scarring may prevent or even delay aortic rupture, which makes the diagnosis even more challenging. However, prompt diagnosis is mandatory since the prognosis of these patients greatly improves with early recognition and treatment.

An aggressive surgical approach together with medical treatment offers good long term results. ${ }^{1}$ In the case of aortic dissection, the medical treatment is designed to control the hypertension and to decrease the $\mathrm{dp} / \mathrm{dt}$ of the arterial pulse with $\beta$ blockers and vasodilators. ${ }^{10}$ However, caution is advised with the use of $\beta$ blockers, since circulating catecholamines appear crucial for the grafted heart to enable exercise performance. Accordingly, $\beta$ blockers should be restricted to patients with drug refractory arterial hypertension.

Mediastinitis after transplantation must be debrided early and followed up carefully with blood cultures, echocardiogram, and thoracic magnetic resonance image. ${ }^{1}$ If the infection is recurrent, immediate surgical intervention is indicated. Whenever the dissection extends to the ascending aorta or whenever a pseudoaneurysm is present, the affected aortic segment must be resected and replaced with a homologous conduit. Long term postoperative administration of antibiotics is mandatory.

In conclusion, we report the first case of recognition and treatment of a subacute type A aortic dissection in a cardiac allograft recipient with dissection of the sinuses of Valsalva, dilatation of the aortic root, and severe aortic regurgitation that was successfully treated with a Bentall procedure.
Although ascending aortic dissection remains rare in cardiac allograft recipients (incidence $<1 \%$ ), ${ }^{3}$ the diagnosis should be considered when dilatation of the aortic arch and aortic regurgitation are present. Unfortunately the diagnosis is often made at necropsy.

\section{Authors' affiliations \\ C Schellemans, W Tack, M Vanderheyden, Cardiovascular Centre, Onze Lieve Vrouwziekenhuis, Aalst, Belgium}

Correspondence to: $\operatorname{Dr} M$ Vanderheyden, Cardiovascular Centre, Onze Lieve Vrouwziekenhuis, Moorselbaan 164, 9400 Aalst, Belgium; marc.vanderheyden@olvz-aalst.be

Accepted 15 April 2004

\section{REFERENCES}

1 Vigano' M, Rinaldi M, D'Armini AM, et al. The spectrum of aortic complications after heart transplantation. Ann Thorac Surg 1999;68:105-8.

2 Derumeaux G, Habib G, Schleifer DM, et al. Standard orthotopic heart transplantation versus total orthotopic heart transplantation: a transesophageal echocardiography study of the incidence of left atrial thrombosis. Circulation 1995;92(9 suppl):H196-201.

3 Sahar G, Stamler A, Erez E, et al. Etiological factors influencing the development of atrioventricular valve incompetence after heart transplantation. Transplant Proc 1997;29:2675-6.

4 De Simone R, Lange R, Sack F-U, et al. Atrioventricular valve insufficiency and atrial geometry after orthotopic heart transplantation. Ann Thorac Surg 1995;60:1686-93.

5 Dreyfus G, Jebara VA, Couetil J, et al. Kinking of the pulmonary artery: a treatable cause of acute right ventricular failure after heart transplantation. $J$ Heart Transplant 1990;9:575-6.

6 Law Y, Bellasario A, West L, et al. Supramitral valve obstruction from hypertrophied native atrial tissue as a complication of orthotopic heart transplantation. J Heart Lung Transplant 1997;16:922-5.

7 Oaks TE, Rayburn BK, Brown ME, et al. Acquired cor triatum after orthotopic cardiac transplantation. Ann Thorac Surg 1995;59:751-3.

8 Laske A, Carrel T, Niederhauser U, et al. Modified operation technique for orthotopic heart transplantation. Eur J Cardiothorac Surg 1995;9:120-6.

9 Pak P, Gillinov AM, Hruban RH, et al. Aortic dissection in a cardiac allograft recipient: a case report. J Heart Lung Transplant 1995;14:1003-5.

10 Defraigne JO, Demoulin JC, Detry O, et al. Successful management of acute aortic dissection in a heart transplant recipient. Acta Chir Belg 1997;97:141-3.

11 Piotrowski JJ, Macintyre KE, Hunter GC, et al. Abdominal aortic aneurysm in the patients undergoing cardiac transplantation. J Vasc Surg 1991;14:460-7.

12 Bull DA, Neumayer LA, Venerus BJ, et al. The effects of improved haemodynamics on aortic dimensions in patients undergoing heart transplantation. J Vasc Surg 1994;20:539-45.

13 Coppola D, Alpern J, Brozena S, et al. Aortic dissection in cardiac allograft recipients: a report of two cases. Arch Pathol Lab Med 1993;117:1170-3. 